\title{
Cross-Border Portfolio Investment from Developing Economies and Top Major Partners, Using the Gravity Model
}

\author{
Sahar Hassan Khayat ${ }^{1}$ \& Samiha Hassan Khayyat ${ }^{1}$ \\ ${ }^{1}$ Department of Economics, Faculty of Economics and Administration, King Abdulaziz University, Jeddah, \\ Saudi Arabia \\ Correspondence: Assistant Professor, Department of Economics, Faculty of Economics and Administration, King \\ Abdulaziz University, P.O. Box 3024, Jeddah 21471, Saudi Arabia. Tel: 966-505-646-027. E-mail: \\ saharkayat@hotmail.com; skhayat@kau.edu.sa
}

Received: September 5, 2018

Accepted: October 27, 2018

Online Published: October 31, 2018

doi:10.5539/ijef.v10n11p137

URL: https://doi.org/10.5539/ijef.v10n11p137

\begin{abstract}
The study has evaluated the volume of cross-border portfolio investment from developing economies and top major partners, using gravity model. Panel data set is used on bilateral gross cross-border investment flows between 37 developing countries and 79 host countries, which are the top five in the world from 2001 and 2012. The positive and significant coefficient on GDP per capita in a destination country can explain a significant part of Lucas paradox. It supported the reason why developing capital is invested outside the region. The results showed statistically insignificant effect of bilateral trade in lagged form on asset holdings. There is a high correlation between GDP per capita in source country and market capitalization of listed companies in the source countries. The significant positive coefficient of GDP per capita of source economies in OLS suggested that richer economies are major sources of portfolio investment Geographical proximity exerts a significant positive influence on the assets that investors may diversify their portfolios.
\end{abstract}

Keywords: investment, portfolio, management, Gravity model

\section{Introduction}

International capital flow which is associated with the purchase of bonds and stocks, without acquiring a controlling stake, is called equity or portfolio investments. The importance of cross-border flows of capital have increased in the recent decades; however, the cross-border flows rose sharply in the early 1990s. Moreover, multinational enterprises were thought to be as tools of imperialism by the underdeveloped countries and were regarded as a persistent threat to their economies (Narula \& Pinel, 2017). During 1990s, capital flows were increased significantly in developing countries as a source of investment with the removal of restrictions on foreign investors' entry into local economies. The volume of cross-border portfolio investment was also increased for both developing and industrial economies, indicating the high degree to which developing countries had become integrated into the global economy.

One of the reasons behind decrease in the foreign investments is the fluctuation of government policies. All foreign direct investments are subjected to risk due to shift in government policies which can affect the payoffs to investors (Julio \& Yook, 2016). Moreover, the constraint in the global banking sector is the contemporaneous fundamental risks which restrict the growth of the global banking sector (Bruno \& Shin, 2014). There is a positive influence of M\&As on the total factor productivity in developed countries (Ashraf, Herzer, \& Nunnenkamp, 2016). Until 1970s, the development impact of FDI was also not discussed in the academic debate. Thus, the reason behind it was portfolio investment, distinguishing theories and the behaviour and existence of multinational enterprises (Narula \& Pinel, 2017). Moreover, there is a need to understand how foreign direct investments affect the host countries. Since MNCs assumed a major role in global investment, trade and production in final and intermediate goods, developing economies are also increasing their share in FDI. To this end, domestic conditions should be improved to improve the complementarity between financial market conditions and FDI (Alfaro \& Chauvin, 2016).

The gravity approach to the international trade in European Community countries was presented by Birula (2015), who depicted that distance is negative as well as significant. This helped in evaluating a new method of describing the location of two trading partners, relative to different countries across the world. The new measure 
was based on the concept of the world trade gravity center and associated with the gravity theory. GDP per capita is positive and significant; however, distance has a negative effect as expected. The volume of cross-border portfolio investment using gravity model has been investigated by limited studies. Therefore, the present study uses gravity model to evaluate volume of cross-border portfolio investment from developing economies and top major partners.

The study has contributed to the literature and gravity model from the period 2001 to 2012 to answer the research questions. Populations for both source and host countries have been positively correlated with portfolio investment clarifying that larger countries export and import portfolio investment as the major source and host countries. The results also showed the effects of market capitalization of listed companies on portfolio investment that were positive and significant for source countries, and had a negative effect in some regressions, which was an unexpected sign, but had positive effects on host countries. The import and export between countries is highly reduced if both the states do not stand on the same ground. Furthermore, it was found that trade in a level had positive effects, and trade in a lagged one-year cycle had an explanatory variable that gave insignificant effects. For instance, USA and oil-rich Persian Gulf countries share strong association that results in deterioration of their relationship with other developed countries with whom they trade.

\section{Literature Review}

Cross-border flow tends to increase the linkages, which appear to propagate information resulting in increase of the global financial integration of firms (Moshirian et al., 2017). However, there is a dramatic increase in the financial flows between the countries as measured in the past decade (Bank for International Settlements, 2010). The bilateral financial flows are restricted through various informational frictions that can be reduced through cross-border migration of people (Coeurdacier \& Rey, 2013). Previous empirical studies have focused on the relationship between a standard gravity model and cross-border mergers acquisitions. For instance, Hattari and Rajan (2011) compared the determinants of different types of capital flows (FDI, equity FPI, and M\&As) using bilateral panel data and a gravity model over the period 2000-2007 (48 sources and 57 host countries). Shin and Yang (2012) also adopted a gravity model to investigate the complementarities between financial assets and trade in assets between 1983 and 2004, which showed that per capita GDP for source and destination countries was statistically significant. In addition, a gravity model did well for the trade impact through distance, border, and common language.

The present study has focused on developing countries as the main rationale. The lack of evidence to explanatory variables applying to developing economies is the main reason to focus on this topic. The financial asset sector has been extensively applied; particularly in developed and emerging markets with developing economies, as a source country (Hattari \& Rajan, 2011; Aggarwal et al., 2012; Peter, 2012; Abid \& Bahloul, 2011; Lee et al., 2012). Choi et al. (2014) and Daly and Vo (2013) conducted a study considering the United States and Australia's financial asset sectors. However, less attention has been paid to financial assets in developing countries (Garcia-Herrero et al., 2009). Lee et al. (2012) focused on the determinants of cross-border portfolio investment among APEC economies, using a gravity model. It showed that APEC members have higher investment between themselves rather than with non-members. Natural logarithm form of per capita GDP of source and destination economies was positive and significant, and suggested that richer economies are the major sources and the major recipients of equity investment. Balli et al. (2011) examined the determinants of cross-border portfolio investment, focusing on the difference between total foreign investment holdings, bond holdings, and debt holdings to the GCC economies, with 35 sources as host countries for the period 2001 to 2006. GDP per capita did not have any significant results in total investment holdings but was positive and significant in bond holdings and debt holdings. It was concluded that trade had a positive sign and was statistically significant to explain a portfolio investment in the GCC region.

Abidin and Sahlan (2013) conducted a study to identify the determinants of export between Malaysia and OIC member countries. Gravity model was used to analyze the effect of several variables on the export of Malaysia. The results estimated from the study and the country's GDP was found highly significant, however, the study showed that the country's GDP was evaluated based on the size of the economy. The country is likely to produce more goods due to the increase in GDP; however, the goods were not exported as required. Similarly, gravity model was also adopted by Shin and Yang (2012) to investigate the complementarities between financial assets and trade in the assets between 1983 and 2004. The results showed significant effect of per capita GDP for source and destination countries, bilateral trade, border and common language. Coeurdacier and Martin (2009) analysed the euro's effects on the determinants of banking assets. The results depicted positive effect of trade and GDP as a proxy for market size, market capitalization, and common language. 
Gravity analysis was used by Elshehawy et al. (2014) to study the factors affecting Egypt's exports. The results obtained from the study were found out such that GDP of the importing countries, GDP of Malaysia, and the population of the importing country posed a positive influence on Egypt's exports. Moreover, these factors had a positive influence on the Egypt's GDP. The exports of Egypt increased as a result of increased GDP. There was a significant effect of distance on the trade flow between the countries; whereas, the distance posed no significant influence on the trade between Egypt and the importing countries. Hattari and Rajan (2011) compared the determinants of different types of capital flows (FDI, equity FPI, and M\&As). The results for the impact of explanatory variables on FDI concluded that the populations of source and host countries and GDP per capita of the host country were positive and significant. A common language and contiguous dummy were positive and significant. On the other hand, the coefficient of distance and GDP per capita of source country was negative and statistically significant.

\subsection{Study Hypotheses}

H0: There is positive impact of market capitalization of companies on portfolio investment, using gravity model.

H1: There is negative impact of market capitalization of companies on portfolio investment, using gravity model.

\section{Methodology}

\subsection{Data Description}

The study used panel data consisting of 37 developing countries investing and 79 receiving from the period 2001 to 2012. Appendix A (Table A1) gives a list of the source countries, and Appendix A (Table A2) a list of the host countries included in each regression. The study considered the aggregate equity securities and debt securities, short term and long term, between source countries $i$ and host country $j$ (Abid \& Bahloul, 2011; Lee et al., 2012; Peter, 2012; Balli et al., 2011). Data was obtained from the International Monetary Fund's (IMF) coordinated Portfolio Investment Survey (CIPS) and converted in real terms using the U.S. GDP deflator (Papaioannou, 2009; Garcia-Herrero et al., 2009). Data for U.S. GDP deflator was also provided by the World Bank (2014) World Development Indicators Database (WDI).

Three estimations, the ordinary least squares (OLS), fixed effects (FE), and random effects (RE) estimations to determinants of portfolio investment assets from the developing countries and the top major partners over the world have been employed. The pooled (OLS) technique has been applied to the study of capital flows, particularly in asset holdings (Choi et al., 2014; Papaioannou, 2009; Aggarwal et al., 2012; Abid \& Bahloul, 2011; Portes \& Rey, 2005), and the fixed effects estimation has been applied in asset holdings (Hattari \& Rajan, 2011; Lee et al., 2012).

\subsection{Gravity Model}

The model specification and econometric methodology were used to study the relationship between the explanatory variables as the main determinants of cross-border portfolio investment in developing countries to answer the research questions. This study is very much related to recent literature that have used a financial gravity equation in their research, particularly Portes et al. (2001), Portes and Rey (2005) and Aviat and Coeurdacier (2007). In order to estimate the effect of explanatory variables on cross-border portfolio investment, a baseline gravity model (equation 1 ) is formulated as:

$$
\begin{gathered}
\text { Ln Asset }_{i j t}=\alpha_{0}+\beta_{1} \operatorname{lnGDP~}_{\text {L }} c_{i t}+\beta_{2} \ln G D P p c_{j t}+\beta_{3} \operatorname{lnPop}_{i t}+\beta_{4} \text { InPop }_{j t}+\beta_{5} \text { lnDistance }_{i j}+ \\
\beta_{6} \text { Contig }_{i j}+\beta_{7} \text { Comonlang }_{i j}+\varepsilon_{i j t}
\end{gathered}
$$

Equation 1 is rewritten to include risk premium, market capitalization of listed companies, bilateral trade in level, and bilateral trade in lagged form, as shown below:

$$
\begin{array}{r}
\text { Ln Asset }_{i j t}=\alpha_{0}+\beta_{1} \text { ln GDP pc }_{i t}+\beta_{2} \text { ln GDPpc }_{j t}+\beta_{3} \text { lnPop }_{i t}+\beta_{4} \text { InPop }_{j t}+\beta_{5} \text { lnDistance }_{i j}+ \\
\beta_{6} \text { Contig }_{i j}+\beta_{7} \text { Comonlang }_{i j}+\beta_{8} \text { Riskprem }_{i t}+\beta_{9} \text { Riskprem }_{j t}+\beta_{10} \text { Mark }_{j t}+\beta_{11} \text { Trade }_{i j t}+\varepsilon_{i j t}
\end{array}
$$

Where, $i$ and $\mathrm{j}$ indicate the "source" and "host" country, respectively; t denotes time (2001-2012).

As pointed out in the preceding sections, the regression results were obtained with year-specific effects, source-country fixed effects, and also included source country year fixed effects to control unobservable country-specific effects invariant over the time. Equation (2) is rewritten to include all those dummies in estimations.

$$
\begin{array}{r}
{\text { Ln } \text { Asset }_{i j t}}=\alpha_{0}+\beta_{1} \text { lnGDP pc }_{i t}+\beta_{2} \operatorname{lnGDPpc}_{j t}+\beta_{3} \text { InPop }_{i t}+\beta_{4} \operatorname{lnPop}_{j t}+\beta_{5} \text { InDistance }_{i j}+ \\
\beta_{6} \text { Contig }_{i j}+\beta_{7} \text { Comonlang }_{i j}+\beta_{8} \text { Riskprem }_{i t}+\beta_{9} \text { Riskprem }_{j t}+\beta_{10} \text { Mark }_{j t}+\beta_{11} \text { Trade }_{i j t}+
\end{array}
$$




$$
\beta_{12} \text { Trade }_{i j t}+\alpha_{t}+\alpha_{i t}+\left[\alpha_{t}+\alpha_{i t}\right]+\varepsilon_{i j t}
$$

Where, $\alpha$ t in regression is a vector of year-specific effects; $\alpha$ it is a vector of source-country fixed effects; $\left[\alpha_{t}+\alpha_{i t}\right]$ are vectors of source country year fixed effects.

\section{Results}

Table 1 presented the descriptive statistics of the data, while table 2 showed a correlation coefficient between the variables and portfolio investment. There is no evidence of multicollinearity, concerning the explanatory variable. The main results are described in tables 3-5. In each table, eight regressions are reported with various combinations of explanatory variables, and estimation methods. Models 1, 5 and 9 show the baseline results obtained from the estimation of equation (1). Models 2 to 4, 6 to 8 and 10 to 12, are the models used as a further set of economic and financial controls from the estimates of equation (2).

Table 1 . Summary statistics on the FPI and the gravity model

\begin{tabular}{|c|c|c|c|c|}
\hline Variable & Mean & Std. Dev & Min & Max \\
\hline RFPI $_{\mathrm{ij}}$ & 2703.506 & 14280.34 & $2.80 \mathrm{e}-06$ & 390241.3 \\
\hline $\operatorname{Ln}_{\text {RFPI }}{ }_{i j}$ & 4.354091 & 3.102632 & -12.78695 & 12.87452 \\
\hline GDP pc ${ }_{i}$ & 8861.53 & 13321.14 & 348.78 & 81531.6 \\
\hline $\operatorname{Ln}_{\text {GDP }} \mathrm{pc}_{\mathrm{i}}$ & 8.399 & 1.170 & 5.854 & 11.308 \\
\hline $\mathrm{GDP}_{\mathrm{pc}}{ }_{\mathrm{j}}$ & 31518.08 & 19149.81 & 249.06 & 87716.73 \\
\hline $\mathrm{n}$ GDP pc ${ }_{j}$ & 9.985 & 1.119 & 5.517 & 11.381 \\
\hline Population $_{i}$ & $9.93 e+07$ & $2.41 e+08$ & 62504 & $1.24 \mathrm{e}+09$ \\
\hline Population $_{i}$ & 16.477 & 2.377 & 11.042 & 20.935 \\
\hline Population $_{\mathrm{j}}$ & $9.83 \mathrm{e}+07$ & $2.14 \mathrm{e}+08$ & 43317 & $1.35 \mathrm{e}+09$ \\
\hline LnPopulation $_{\mathrm{j}}$ & 16.681 & 2.413 & 10.676 & 21.023 \\
\hline Market $_{i}$ & 61.405 & 70.032 & 0.334 & 606.001 \\
\hline Market $_{j}$ & 98.290 & 79.661 & 0.334 & 606.001 \\
\hline Risk $_{i}$ & 6.867 & 8.641 & -2.916 & 44.978 \\
\hline Risk $_{j}$ & 4.749 & 8.002 & -7.051 & 53.083 \\
\hline Contig & 0.053 & 0.224 & 0 & 1 \\
\hline Comlang & 0.265 & 0.441 & 0 & 1 \\
\hline Dist & 6667.59 & 4547.63 & 60.770 & 19217.88 \\
\hline Ln Dist & 8.445 & 1.005 & 4.107 & 9.863 \\
\hline Trade $_{\mathrm{ij}}$ & 0.0117 & 0.0412 & 0 & 0.564 \\
\hline TRADE_ij (lagged) & 0.0117 & 0.0412 & 0 & 0.564 \\
\hline
\end{tabular}

Note. All variables are defined in the methodology (Ln meaning in natural logarithm form).

Ln (natural logarithm form).

The average FPI between countries' natural logarithm is $4.35 \%$ with an overall standard deviation of $3.10 \%$. The average GDP pc $i$ natural logarithm is $8.39 \%$ with an overall standard deviation of $1.17 \%$. The average GDP pc $j$ in natural logarithm is $9.98 \%$, and the average natural logarithm of population $\mathrm{i}$ is $16.47 \%$, and the average natural logarithm of population $j$ is $16.68 \%$. Summary statistics for other control variables are presented in Table 2. There is a high correlation between GDP per capita in source country and market capitalization of listed companies in the source countries.

Table 2. Correlation coefficient matrix

\begin{tabular}{|c|c|c|c|c|c|c|c|c|c|c|c|c|c|}
\hline & OLS & RE & RE & RE & RE & FE & FE & FE & FE & OLS & OLS & OLS & OLS \\
\hline Ln GDP pc _i & 1 & & & & & & & & & & & & \\
\hline Ln GDP pc $\_j$ & 0.53 & 1 & & & & & & & & & & & \\
\hline Ln POP_i & 0.15 & 0.04 & 1 & & & & & & & & & & \\
\hline Ln POP_j & -0.16 & -0.43 & -0.01 & 1 & & & & & & & & & \\
\hline RISK_i & 0.19 & 0.08 & 0.31 & -0.05 & 1 & & & & & & & & \\
\hline RISK_j & -0.05 & $0.07-$ & 0.02 & 0.45 & 0.01 & 1 & & & & & & & \\
\hline
\end{tabular}




\begin{tabular}{ccccccccccccc}
\hline MARK_i & -0.08 & -0.07 & -0.46 & 0.04 & 0.16 & -0.06 & 1 & & & & & \\
MARK_j & 0.55 & 0.64 & 0.15 & -0.22 & 0.09 & -0.10 & -0.12 & 1 & & & & \\
TRADE_ij & 0.01 & 0.07 & 0.20 & 0.03 & -0.16 & -0.09 & -0.11 & 0.16 & 1 & & & \\
TRADE_ij (lagged) & 0.02 & 0.22 & -0.24 & -0.08 & -0.21 & -0.07 & 0.01 & 0.19 & 0.25 & 1 & & \\
Ln DIST & 0.26 & 0.10 & 0.33 & 0.08 & 0.54 & 0.08 & 0.04 & 0.23 & -0.19 & -0.34 & 1 & \\
CONTIG & 0.03 & 0.13 & -0.10 & 0.04 & -0.03 & 0.01 & -0.01 & 0.07 & 0.02 & 0.69 & -0.26 & 1 \\
COMLANG & 0.32 & 0.35 & -0.09 & $0.19-$ & -0.13 & -0.19 & -0.10 & 0.45 & 0.28 & 0.27 & -0.07 & 0.11 \\
\hline
\end{tabular}

\subsection{Determinants of Cross-Border Portfolio Investment}

The results showed that GDP per capita for source countries had a positive effect on cross-border investment and significance for all three methods. The significant positive coefficient of GDP per capita of source economies in OLS (models 1 to 4), FE (models 5 to 8), and RE (models 9 to 12) suggested that richer economies are major sources of portfolio investment.

Table 3. Determinants of cross-border portfolio investment

\begin{tabular}{|c|c|c|c|c|c|c|c|c|c|c|c|c|}
\hline Regressor & OLS & OLS & OLS & OLS & FE & FE & FE & FE & RE & RE & RE & RE \\
\hline & (1) & (2) & (3) & (4) & (5) & (6) & (7) & (8) & (9) & (10) & (11) & (12) \\
\hline Ln GDP pc & $1.513^{* * *}$ & $1.318^{* * * *}$ & $1.259^{* * *}$ & $1.327 * * *$ & $4.119^{* * * *}$ & $4.575^{* * *}$ & $4.623^{* * *}$ & $4.437 * * *$ & $2.534 * * *$ & $2.792^{* * *}$ & $2.675^{* * * *}$ & $2.786^{* * *}$ \\
\hline$\_\mathrm{i}$ & $(0.000)$ & $(0.000)$ & $(0.000)$ & $(0.000)$ & $(0.000)$ & $(0.000)$ & $(0.000)$ & $(0.000)$ & $(0.000)$ & $(0.000)$ & $(0.000)$ & $(0.000)$ \\
\hline Ln GDP pc_ & $0.693 * * *$ & $0.717 * * *$ & $0.781^{* * *}$ & $0.641 * * *$ & $1.559 * *$ & 0.595 & 0.985 & 1.123 & $0.927 * * *$ & $0.954 * * *$ & $0.957 * * *$ & $0.939^{* * *}$ \\
\hline $\mathrm{j}$ & $(0.000)$ & $(0.000)$ & $(0.000)$ & $(0.000)$ & $(0.015)$ & $(0.732)$ & $(0.596)$ & $(0.544)$ & $(0.000)$ & $(0.002)$ & $(0.001)$ & $(0.006)$ \\
\hline \multirow{2}{*}{$\mathrm{Ln}_{\mathrm{POP}} \mathrm{i}$} & $0.246^{* * * *}$ & $0.286^{* * * *}$ & $0.265^{* * *} *$ & $0.280^{* * * *}$ & $3.744 * * *$ & 0.790 & 0.753 & 1.242 & $0.625 * * *$ & $0.846^{* * *} *$ & $0.794 * * *$ & $0.828^{* * *} *$ \\
\hline & $(0.000)$ & $(0.000)$ & $(0.000)$ & $(0.000)$ & $(0.001)$ & $(0.448)$ & $(0.473)$ & $(0.226)$ & $(0.000)$ & $(0.000)$ & $(0.000)$ & $(0.000)$ \\
\hline \multirow[t]{2}{*}{$\operatorname{Ln}_{\mathrm{POP}} \mathrm{j}$} & $0.342 * * *$ & $0.686^{* * * *}$ & $0.701 * * *$ & $0.0662 * * *$ & $1.333^{* *}$ & $7.509 * * *$ & $7.610^{* * *}$ & $6.990 * * *$ & $0.462 * * *$ & $0.704 * * *$ & $0.721 * * *$ & $0.701^{* * *}$ \\
\hline & $(0.000)$ & $(0.000)$ & $(0.000)$ & $(0.000)$ & $(0.034)$ & $(0.000)$ & $(0.000)$ & $(0.000)$ & $(0.000)$ & $(0.000)$ & $(0.000)$ & $(0.000)$ \\
\hline \multirow[t]{2}{*}{ RISK_i } & & $-0.019 * *$ & $-0.017 * *$ & $-0.020 * *$ & & $0.048^{* *}$ & $0.051^{* *}$ & $0.051^{* * *}$ & & $-0.0213^{*}$ & $-0.020^{*}$ & -0.015 \\
\hline & & $(0.024)$ & $(0.043)$ & $(0.033)$ & & $(0.034)$ & $(0.028)$ & $(0.036)$ & & $(0.092)$ & $(0.099)$ & $(0.270)$ \\
\hline \multirow[t]{2}{*}{ RISK_j } & & 0.0017 & 0.018 & 0.012 & & 0.011 & 0.015 & 0.005 & & 0.006 & 0.004 & -0.002 \\
\hline & & $(0.253)$ & $(0.245)$ & $(0.461)$ & & $(0.859)$ & $(0.819)$ & $(0.939)$ & & $(0.889)$ & $(0.925)$ & $(0.951)$ \\
\hline \multirow[t]{2}{*}{ MARK_i } & & $0.009^{* * *}$ & $0.008 * * *$ & $0.009^{* * *}$ & & $-0.002 *$ & $-0.002 *$ & $-0.002^{*}$ & & 0.001 & 0.001 & 0.001 \\
\hline & & $(0.000)$ & $(0.000)$ & $(0.000)$ & & $(0.092)$ & $(0.057)$ & $(0.094)$ & & $(0.304)$ & $(0.250)$ & $(0.304)$ \\
\hline \multirow[t]{2}{*}{ MARK_j } & & $0.002 * * *$ & $0.001^{* *}$ & $0.002 * *$ & & -0.001 & 0.001 & -0.001 & & -0.002 & -0.002 & -0.002 \\
\hline & & $(0.005)$ & $(0.047)$ & $(0.030)$ & & $(0.521)$ & $(0.611)$ & $(0.633)$ & & $(0.171)$ & $(0.108)$ & $(0.210)$ \\
\hline \multirow[t]{2}{*}{ TRADE_ij } & & & $5.967 * * *$ & & & & -6.356 & & & & $6.575^{*}$ & \\
\hline & & & $(0.000)$ & & & & $(0.337)$ & & & & $(0.077)$ & \\
\hline TRADE_ij & & & & -1.472 & & & & -4.098 & & & & 1.245 \\
\hline (lagged) & & & & $(0.358)$ & & & & $(0.584)$ & & & & $(0.665)$ \\
\hline \multirow[t]{2}{*}{ Ln DIST } & $0.154 * * *$ & -0.025 & 0.043 & 0.055 & & & & & 0.068 & -0.140 & -0.055 & -0.057 \\
\hline & $(0.003)$ & $(0.829)$ & $(0.720)$ & $(0.659)$ & & & & & $(0.661)$ & $(0.683)$ & $(0.871)$ & $(0.874)$ \\
\hline \multirow[t]{2}{*}{ CONTIG } & 0.213 & $1.584 * * *$ & 0.609 & $1.688^{* * * *}$ & & & & & -0.175 & 0.287 & -0.426 & 0.456 \\
\hline & $(0.297)$ & $(0.000)$ & $(0.106)$ & $(0.000)$ & & & & & $(0.757)$ & $(0.703)$ & $(0.561)$ & $(0.570)$ \\
\hline \multirow[t]{2}{*}{ COMLANG } & $1.232 * * *$ & $1.282^{* * * *}$ & $1.253^{* * *}$ & $1.222 * * *$ & & & & & $1.248^{* * * *}$ & $1.895 * * *$ & $1.797 * * * *$ & $1.797 * * *$ \\
\hline & $(0.000)$ & $(0.000)$ & $(0.000)$ & $(0.000)$ & & & & & $(0.000)$ & $(0.000)$ & $(0.000)$ & $(0.000)$ \\
\hline \multirow[t]{2}{*}{ CONSTANT } & $-27.165^{* * *}$ & $-31.966 * * *$ & $-32.550 * * *$ & $-31.313^{* * *}$ & $-131.38 * * *$ & $-185.32 * * *$ & $-190.75^{* * *}$ & $-188.27 * * *$ & $-45.806^{* * *}$ & $-54.564 * * *$ & $-53.776^{* * *}$ & $-54.72 * * *$ \\
\hline & $(0.000)$ & $(0.000)$ & $(0.000)$ & $(0.000)$ & $(0.000)$ & $(0.000)$ & $(0.000)$ & $(0.000)$ & $(0.000)$ & $(0.000)$ & $(0.000)$ & $(0.000)$ \\
\hline Number of & 3530 & 838 & 838 & 794 & 3530 & 838 & 838 & 794 & 3530 & 838 & 838 & 794 \\
\hline \multicolumn{13}{|l|}{ Observations } \\
\hline Number of & 375 & 115 & 115 & 109 & 375 & 115 & 115 & 109 & 375 & 115 & 115 & 109 \\
\hline Country & & & & & & & & & & & & \\
\hline $\mathrm{R}^{2}$ (within) & 0.3311 & 0.6221 & 0.6271 & 0.6132 & 0.2395 & 0.3337 & 0.3354 & 0.3272 & 0.2293 & 0.2715 & 0.2642 & 0.2715 \\
\hline $\mathrm{R}^{2}$ (between) & & & & & 0.0284 & 0.2311 & 0.2271 & 0.2050 & 0.3420 & 0.5202 & 0.5332 & 0.5098 \\
\hline $\mathrm{R}^{2}$ (overall) & & & & & 0.0218 & 0.2553 & 0.2491 & 0.2371 & 0.3162 & 0.5622 & 0.5737 & 0.5543 \\
\hline
\end{tabular}

Note. subscript "i" stands for source economy and " $\mathrm{j}$ ” for destination economy.

p-value in parentheses, *significant at 10\%, **significant at 5\%, ***significant at 1\%. Note: Ln (natural logarithm form). 
The coefficient of population of source economies has expected sign, which was positive and statistically significant in OLS (models 1 to 4), FE (is only significant in model 5), and RE in (models 9 to 12). Finally, bilateral trade between source and destination countries relative to the destination country's GDP (models 3, 7 and 11) were added. The coefficient of bilateral trade was positive and significant in both OLS and RE regressions, suggesting that portfolio investment by developing members is greater and with which they enjoy greater trade integration. In addition, the positive effect proposes that portfolio investments were more likely between countries that traded more. Although, GDP per capita of source economies is significant and positive in RE and OLS estimations, it has negative effect as it estimates OLS for destination countries

\subsection{Determinants of Cross-Border Portfolio Investment using Year Dummy Effects}

The study aimed to take certain factors into account; for example, the world business cycle and the global capital market shock. Hence, the results obtained with year-specific effects for foreign portfolio investment holdings are reported in table 4 and using the equation (3).

Table 4. Determinants of cross-border portfolio investment with year dummy effects

\begin{tabular}{|c|c|c|c|c|c|c|c|c|c|c|c|c|}
\hline Regressor & OLS & OLS & OLS & OLS & FE & FE & FE & FE & RE & RE & RE & $\mathbf{R E}$ \\
\hline & (1) & (2) & (3) & (4) & (5) & (6) & (7) & (8) & (9) & (10) & (11) & (12) \\
\hline \multirow[t]{2}{*}{ Ln GDP pc _i } & $1.408^{* * *}$ & $1.057 * * *$ & $1.001 * * *$ & $1.044 * * *$ & 0.461 & -0.730 & -0.725 & -0.798 & $1.310^{* * *}$ & $1.459 * * *$ & $1.375^{* * *}$ & $1.384 * * *$ \\
\hline & $(0.000)$ & $(0.000)$ & $(0.000)$ & $(0.000)$ & $(0.396)$ & $(0.622)$ & $(0.628)$ & $(0.605)$ & $(0.000)$ & $(0.000)$ & $(0.000)$ & $(0.000)$ \\
\hline \multirow[t]{2}{*}{ Ln GDP pc $c_{-} j$} & $0.654 * * *$ & $0.714 * * *$ & $0.783^{*} * *$ & $0.641 * * *$ & 0.900 & 1.297 & 1.306 & 1.735 & $0.540 * * *$ & $0.664^{* *}$ & $0.673^{* *}$ & $0.663^{*}$ \\
\hline & $(0.000)$ & $(0.000)$ & $(0.000)$ & $(0.000)$ & $(0.146)$ & $(0.477)$ & $(0.495)$ & $(0.373)$ & $(0.001)$ & $(0.035)$ & $(0.028)$ & $(0.073)$ \\
\hline \multirow[t]{3}{*}{$\mathrm{Ln}_{\mathrm{POP}} \mathrm{i}^{\mathrm{i}}$} & $0.186^{* * * *}$ & $0.164 * *$ & $0.145^{* *}$ & $0.151^{* *}$ & $-2.377 * *$ & $-5.052 * *$ & $-5.048 * *$ & $-4.519^{* *}$ & $0.132 * *$ & 0.196 & 0.160 & 0.151 \\
\hline & $(0.000)$ & $(0.016)$ & $(0.033)$ & $(0.041)$ & $(0.026)$ & $*$ & $*$ & $(0.013)$ & $(0.048)$ & $(0.178)$ & $(0.281)$ & $(0.328)$ \\
\hline & & & & & & $(0.004)$ & (0.004) & & & & & \\
\hline \multirow[t]{2}{*}{ Ln POP_j } & $0.331^{* * *} *$ & $0.688 * * *$ & $0.704 * * *$ & $0.673 * * *$ & $0.898^{* *} *$ & $5.758 * * *$ & $5.762 * * *$ & $5.280^{* *}$ & $0.302 * * *$ & $0.617 * * *$ & $0.634 * * *$ & $0.609 * * *$ \\
\hline & $(0.000)$ & $(0.000)$ & $(0.000)$ & $(0.000)$ & $(0.034)$ & $(0.004)$ & $(0.004)$ & $(0.014)$ & $(0.000)$ & $(0.000)$ & $(0.000)$ & $(0.001)$ \\
\hline \multirow[t]{2}{*}{ RISK_i } & & -0.006 & -0.004 & $-0.007^{* *}$ & & 0.032 & 0.032 & 0.035 & & 0.012 & 0.012 & 0.017 \\
\hline & & $(0.457)$ & $(0.595)$ & $(0.430)$ & & (0.193) & (0.195) & $(0.184)$ & & $(0.390)$ & $(0.394)$ & $(0.262)$ \\
\hline \multirow[t]{2}{*}{$R I S K \_j$} & & 0.016 & 0.016 & 0.010 & & -0.026 & -0.026 & -0.033 & & -0.011 & -0.013 & -0.016 \\
\hline & & $(0.294)$ & $(0.280)$ & $(0.500)$ & & $(0.691)$ & $(0.692)$ & $(0.637)$ & & $(0.764)$ & $(0.730)$ & $(0.697)$ \\
\hline \multirow[t]{2}{*}{ MARK_i } & & $0.010^{* * *}$ & $0.010^{* * *}$ & $0.010 * * *$ & & $-0.002 *$ & $-0.002^{*}$ & $-0.002 *$ & & 0.001 & 0.002 & 0.001 \\
\hline & & $(0.000)$ & $(0.000)$ & $(0.000)$ & & $(0.068)$ & $(0.068)$ & $(0.094)$ & & $(0.178)$ & $(0.143)$ & $(0.230)$ \\
\hline \multirow[t]{2}{*}{ MARK_j } & & $0.003 * * *$ & $0.002 * * *$ & $0.003 * * *$ & & -0.001 & -0.001 & -0.001 & & -0.001 & -0.002 & 0.001 \\
\hline & & $(0.000)$ & $(0.004)$ & $(0.001)$ & & $(0.656)$ & $(0.661)$ & $(0.946)$ & & $(0.893)$ & $(0.734)$ & $(0.867)$ \\
\hline \multirow[t]{2}{*}{ TRADE_ij } & & & $6.048 * * *$ & & & & -0.139 & & & & $5.901 *$ & \\
\hline & & & $(0.000)$ & & & & (0.976) & & & & $(0.091)$ & \\
\hline \multirow[t]{2}{*}{ TRADE_ij (lagged) } & & & & -0.811 & & & & 1.290 & & & & 3.907 \\
\hline & & & & $(0.589)$ & & & & $(0.872)$ & & & & $(0.182)$ \\
\hline \multirow[t]{2}{*}{ Ln DIST } & $0.1855^{* * * *}$ & -0.026 & 0.040 & 0.040 & & & & & 0.197 & 0.178 & 0.248 & 0.202 \\
\hline & $(0.000)$ & $(0.810)$ & $(0.718)$ & $(0.737)$ & & & & & $(0.157)$ & $(0.559)$ & $(0.415)$ & $(0.531)$ \\
\hline \multirow[t]{2}{*}{ CONTIG } & 0.291 & $1.716^{* * * *}$ & $0.721 *$ & $1.806^{* * *}$ & & & & & 0.084 & $1.634 *$ & 0.970 & $1.742 * *$ \\
\hline & $(0.137)$ & $(0.000)$ & $(0.052)$ & $(0.000)$ & & & & & $(0.876)$ & $(0.041)$ & $(0.209)$ & $(0.037)$ \\
\hline \multirow[t]{2}{*}{ COMLANG } & $1.238^{* * * *}$ & $1.161^{* * *}$ & $1.137 * * *$ & $1.149^{* * *}$ & & & & & $1.098 * * *$ & $1.666^{* * *}$ & $1.577 * * *$ & $1.680 * * *$ \\
\hline & $(0.000)$ & $(0.000)$ & $(0.000)$ & $(0.000)$ & & & & & $(0.000)$ & $(0.001)$ & $(0.002)$ & $(0.002)$ \\
\hline \multirow[t]{2}{*}{ CONSTANT } & $-25.498 * * *$ & $-27.400 * * *$ & $-28.098^{* * * *}$ & $-27.643^{* * *}$ & -14.390 & -18.625 & -18.876 & -23.310 & -21.074 & $-30.308^{* * *}$ & $-29.992 * * *$ & $-29.117 * * *$ \\
\hline & $(0.000)$ & $(0.000)$ & $(0.000)$ & $(0.000)$ & $(0.442)$ & $(0.698)$ & $(0.703)$ & $(0.654)$ & $(0.000)$ & $(0.000)$ & $(0.000)$ & $(0.000)$ \\
\hline Year effects & Yes & Yes & Yes & Yes & Yes & Yes & Yes & Yes & Yes & Yes & Yes & Yes \\
\hline Number of & 3530 & 838 & 838 & 794 & 3530 & 838 & 838 & 794 & 3530 & 838 & 838 & 794 \\
\hline \multicolumn{13}{|l|}{ Observations } \\
\hline Number of Country & 375 & 115 & 115 & 109 & 375 & 115 & 115 & 109 & 375 & 115 & 115 & 109 \\
\hline $\mathrm{R}^{2}$ (within) & 0.3894 & 0.6655 & 0.6706 & 0.6567 & 0.3432 & 0.4293 & 0.4293 & 0.4253 & 0.3385 & 0.3943 & 0.3935 & 0.3966 \\
\hline $\mathrm{R}^{2}$ (between) & & & & & 0.1060 & 0.1624 & 0.1624 & 0.1543 & 0.3631 & 0.5786 & 0.5932 & 0.5696 \\
\hline $\mathrm{R}^{2}$ (overall) & & & & & 0.1333 & 0.1689 & 0.1690 & 0.1552 & 0.3867 & 0.6151 & 0.6263 & 0.6083 \\
\hline
\end{tabular}

Note. subscript "i" stands for source economy and " $\mathrm{j}$ " for destination economy.

p-value in parentheses, *significant at 10\%, **significant at 5\%, ***significant at $1 \%$. Note: Ln (natural logarithm form). 


\subsection{Determinants of Cross-Border Portfolio Investment using Source Country Fixed Effects}

The estimation results for equation (3) including dummy are shown in table 5 and table 6 . The effect of GDP per capita for source and destination countries was same as expected. GDP per capita for source countries was positive and significant for all methods. The coefficients of GDP per capita for destination countries are positive and significant in both OLS regression (models 1 to 4) and RE (models 9 to 12), while in FE estimation (only model 5). The coefficient on population of source economies has the expected sign, which is positive and statistically significant in OLS (models 1 to 4), FE (is only significant in model 5), and RE (models 9 to 12).

Table 5. Determinants of cross-border portfolio investment with source country effects

\begin{tabular}{|c|c|c|c|c|c|c|c|c|c|c|c|c|}
\hline \multirow[t]{2}{*}{ Regressor } & \multirow{2}{*}{$\frac{\text { OLS }}{(1)}$} & \multirow{2}{*}{$\frac{\text { OLS }}{(2)}$} & \multirow{2}{*}{$\frac{\text { OLS }}{(3)}$} & \multirow{2}{*}{$\frac{\text { OLS }}{(4)}$} & FE & FE & FE & FE & RE & \multirow{2}{*}{$\frac{\text { RE }}{(10)}$} & \multirow{2}{*}{$\frac{\text { RE }}{(11)}$} & \multirow{2}{*}{$\frac{\mathrm{RE}}{(12)}$} \\
\hline & & & & & (5) & (6) & (7) & (8) & (9) & & & \\
\hline \multirow[t]{2}{*}{ Ln GDP pc _i } & $\mathrm{i} 4.520 * * *$ & $6.276 * * *$ & $6.360 * * *$ & $6.316^{* * *}$ & $4.119^{* * *}$ & $4.575^{* * *}$ & $4.623 * * *$ & $4.437 * * *$ & $4.508^{* * *}$ & $5.823 * * *$ & $5.893 * * *$ & $5.702 * * *$ \\
\hline & $(0.000)$ & $(0.000)$ & $(0.000)$ & $(0.000)$ & $(0.000)$ & $(0.000)$ & $(0.000)$ & $(0.000)$ & $(0.000)$ & $(0.000)$ & $(0.000)$ & $(0.000)$ \\
\hline \multirow{2}{*}{ Ln GDP pc_j } & $\mathrm{j} 0.732 * * *$ & $0.929 * * *$ & $0.913 * * *$ & $0.870^{* * *}$ & $1.559 * *$ & 0.595 & 0.985 & 1.123 & $0.660 * * *$ & $0.792 * * *$ & $0.785 * * *$ & $0.791 * *$ \\
\hline & $(0.000)$ & $(0.000)$ & $(0.000)$ & $(0.000)$ & $(0.015)$ & $(0.732)$ & $(0.596)$ & $(0.544)$ & $(0.000)$ & $(0.003)$ & $(0.004)$ & $(0.013)$ \\
\hline \multirow[t]{2}{*}{ Ln POP_i } & $4.319 * * *$ & $2.860 * * *$ & $2.902 * * *$ & $2.471 * * *$ & $3.744 * * *$ & 0.790 & 0.753 & 1.242 & $4.492 * * *$ & $3.035 * * *$ & $3.082 * * *$ & $3.244 * * *$ \\
\hline & $(0.000)$ & $(0.000)$ & $(0.000)$ & $(0.004)$ & $(0.001)$ & $(0.448)$ & $(0.473)$ & $(0.226)$ & $(0.000)$ & $(0.004)$ & $(0.004)$ & $(0.002)$ \\
\hline \multirow[t]{2}{*}{ Ln POP $\_j$} & $0.324 * * *$ & $0.781 * * *$ & $0.779 * * *$ & $0.800^{* * * *}$ & $1.333^{* * *}$ & $7.509^{* * *}$ & $7.610^{* * * *}$ & $6.990 * * *$ & $0.301 * * *$ & $0.774 * * *$ & $0.774 * * *$ & $0.787 * * *$ \\
\hline & $(0.000)$ & $(0.000)$ & $(0.000)$ & $(0.000)$ & $(0.034)$ & $(0.000)$ & $(0.000)$ & $(0.000)$ & $(0.000)$ & $(0.000)$ & $(0.000)$ & $(0.000)$ \\
\hline \multirow[t]{2}{*}{ RISK_i } & & $0.053 * *$ & $0.055^{* *}$ & $0.052 * *$ & & 0.011 & $0.051^{* *}$ & $0.051^{* *}$ & & $0.046^{* *}$ & $0.047^{* *}$ & $0.048^{* *}$ \\
\hline & & $(0.030)$ & $(0.023)$ & $(0.040)$ & & $(0.859)$ & $(0.028)$ & $(0.036)$ & & $(0.037)$ & $(0.033)$ & $(0.037)$ \\
\hline \multirow[t]{2}{*}{ RISK_j } & & 0.023 & 0.024 & 0.018 & & $0.048 * *$ & 0.015 & 0.005 & & 0.001 & 0.007 & 0.004 \\
\hline & & $(0.122)$ & $(0.112)$ & $(0.234)$ & & $(0.034)$ & $(0.819)$ & $(0.939)$ & & $(0.825)$ & $(0.812)$ & $(0.910)$ \\
\hline \multirow[t]{2}{*}{ MARK_i } & & $0.003 * *$ & $-0.003^{* *}$ & $-0.003 * *$ & & $-0.002 *$ & $-0.002 *$ & $-0.002 *$ & & -0.002 & $-0.002 *$ & -0.002 \\
\hline & & $(0.034)$ & $(0.026)$ & $(0.033)$ & & $(0.092)$ & $(0.057)$ & $(0.094)$ & & $(0.102)$ & $(0.079)$ & $(0.138)$ \\
\hline \multirow[t]{2}{*}{ MARK_j } & & 0.001 & $0.001^{*}$ & $0.001^{*}$ & & -0.001 & -0.001 & -0.001 & & -0.001 & -0.001 & -0.001 \\
\hline & & $(0.109)$ & $(0.074)$ & $(0.082)$ & & $(0.521)$ & $(0.611)$ & $(0.633)$ & & $(0.310)$ & $(0.378)$ & $(0.508)$ \\
\hline \multirow[t]{2}{*}{ TRADE_ij } & & & -2.505 & & & & -6.356 & & & & -2.510 & \\
\hline & & & $(0.112)$ & & & & $(0.337)$ & & & & $(0.535)$ & \\
\hline \multicolumn{2}{|l|}{ TRADE_ij } & & & -0.724 & & & & -4.098 & & & & -0.389 \\
\hline \multicolumn{2}{|l|}{ (lagged) } & & & $(0.552)$ & & & & $(0.584)$ & & & & $(0.869)$ \\
\hline \multirow[t]{2}{*}{ Ln DIST } & $-0.466^{* * *}$ & $-0.560^{* * *}$ & $-0.607 * * *$ & $-0.539 * * *$ & & & & & $-0.437 * * *$ & $-0.561^{*}$ & $-0.613^{*}$ & -0.531 \\
\hline & $(0.000)$ & $(0.000)$ & $(0.000)$ & $(0.000)$ & & & & & $(0.000)$ & $(0.067)$ & $(0.056)$ & $(0.115)$ \\
\hline CONTIG & -0.236 & $0.820 * *$ & $1.193^{* *}$ & $0.771^{*}$ & & & & & -0.398 & 0.768 & 1.031 & 0.723 \\
\hline & $(0.192)$ & $(0.032)$ & $(0.012)$ & $(0.051)$ & & & & & $(0.303)$ & $(0.368)$ & $(0.266)$ & $(0.410)$ \\
\hline COMLANG & 0.121 & $0.612 * * *$ & $0.616^{* * *}$ & $0.687 * * *$ & & & & & 0.183 & 0.687 & 0.701 & 0.642 \\
\hline & $(0.222)$ & $(0.002)$ & $(0.002)$ & $(0.003)$ & & & & & $(0.390)$ & $(0.114)$ & $(0.106)$ & $(0.187)$ \\
\hline CONSTANT & $-117.72^{* * *}$ & $-107.91 * * *$ & $-108.66^{* * *}$ & $-106.34 * * *$ & $-131.38^{* * *}$ & $-185.32 * * *$ & $-190.75^{* * *}$ & $-188.27 * * *$ & $-122.02 * * *$ & $-109.84^{* * *}$ & $-110.62^{* * *}$ & $-112.45^{* * *}$ \\
\hline & $(0.000)$ & $(0.000)$ & $(0.000)$ & $(0.000)$ & $(0.000)$ & $(0.000)$ & $(0.000)$ & $(0.000)$ & $(0.000)$ & $(0.000)$ & $(0.000)$ & $(0.000)$ \\
\hline $\begin{array}{l}\text { Source } \\
\text { country } \\
\text { effects }\end{array}$ & Yes & Yes & Yes & Yes & Yes & Yes & Yes & Yes & Yes & Yes & Yes & Yes \\
\hline $\begin{array}{l}\text { Number of } \\
\text { Observations }\end{array}$ & 3530 & 838 & 838 & 794 & 3530 & 838 & 838 & 794 & 3530 & 838 & 838 & 794 \\
\hline $\begin{array}{l}\text { Number of } \\
\text { Country }\end{array}$ & 375 & 115 & 115 & 109 & 375 & 115 & 115 & 109 & 375 & 115 & 115 & 109 \\
\hline $\mathrm{R}^{2}$ (within) & 0.5972 & 0.7522 & 0.7528 & 0.7468 & 0.2395 & 0.3337 & 0.3354 & 0.3272 & 0.2344 & 0.2963 & 0.2972 & 0.2965 \\
\hline $\mathrm{R}^{2}$ (between) & & & & & 0.0284 & 0.2311 & 0.2271 & 0.2050 & 0.7088 & 0.7967 & 0.7958 & 0.7915 \\
\hline $\mathrm{R}^{2}$ (overall) & & & & & 0.0218 & 0.2553 & 0.2491 & 0.2371 & 0.5948 & 0.7424 & 0.7431 & 0.7375 \\
\hline
\end{tabular}

Note. subscript "i”" stands for source economy and "j" for destination economy.

p-value in parentheses, *significant at $10 \%$, **significant at 5\%, ***significant at $1 \%$. Note: Ln (natural logarithm form). 
Table 6. Determinants of cross-border portfolio investment with source country year effects

\begin{tabular}{|c|c|c|c|c|c|c|c|c|c|c|c|c|}
\hline Regressor & $\begin{array}{l}\text { OLS } \\
(1)\end{array}$ & $\begin{array}{l}\text { OLS } \\
(2)\end{array}$ & $\begin{array}{l}\text { OLS } \\
(3)\end{array}$ & $\begin{array}{l}\text { OLS } \\
(4)\end{array}$ & $\begin{array}{l}\text { FE } \\
(5)\end{array}$ & $\begin{array}{l}\text { FE } \\
(6)\end{array}$ & $\begin{array}{l}\text { FE } \\
(7)\end{array}$ & $\begin{array}{l}\text { FE } \\
(8)\end{array}$ & $\begin{array}{l}\text { RE } \\
(9) \\
\end{array}$ & $\begin{array}{l}\text { RE } \\
(10)\end{array}$ & $\begin{array}{l}\text { RE } \\
\text { (11) }\end{array}$ & $\begin{array}{l}\text { RE } \\
\text { (12) }\end{array}$ \\
\hline Ln GDP pc _i & $\begin{array}{l}0.640 \\
(0.159)\end{array}$ & $\begin{array}{l}0.304 \\
(0.838)\end{array}$ & $\begin{array}{l}0.395 \\
(0.790)\end{array}$ & $\begin{array}{l}0.518 \\
(0.739)\end{array}$ & $\begin{array}{l}0.461 \\
(0.396)\end{array}$ & $\begin{array}{l}-0.730 \\
(0.622)\end{array}$ & $\begin{array}{l}-0.725 \\
(0.628)\end{array}$ & $\begin{array}{l}-0.798 \\
(0.605)\end{array}$ & $\begin{array}{l}0.491 \\
(0.360)\end{array}$ & $\begin{array}{l}-0.709 \\
(0.615)\end{array}$ & $\begin{array}{l}-0.757 \\
(0.597)\end{array}$ & $\begin{array}{l}-0.662 \\
(0.651)\end{array}$ \\
\hline Ln GDP pc_j & $\begin{array}{l}0.729 * * * \\
(0.000)\end{array}$ & $\begin{array}{l}0.935^{* * * *} \\
(0.000)\end{array}$ & $\begin{array}{l}0.924 * * * \\
(0.000)\end{array}$ & $\begin{array}{l}0.877^{* * *} \\
(0.000)\end{array}$ & $\begin{array}{l}0.900 \\
(0.146)\end{array}$ & $\begin{array}{l}1.297 \\
(0.477)\end{array}$ & $\begin{array}{l}1.306 \\
(0.495)\end{array}$ & $\begin{array}{l}1.735 \\
(0.373)\end{array}$ & $\begin{array}{l}0.620^{* * *} \\
(0.000)\end{array}$ & $\begin{array}{l}0.768^{* * *} \\
(0.005)\end{array}$ & $\begin{array}{l}0.769^{* * *} \\
(0.005)\end{array}$ & $\begin{array}{l}0.775^{* *} \\
(0.015)\end{array}$ \\
\hline Ln POP_i & $\begin{array}{l}-2.185^{* * *} \\
(0.005)\end{array}$ & $\begin{array}{l}-3.626^{* *} \\
(0.020)\end{array}$ & $\begin{array}{l}-3.562^{* *} \\
(0.022)\end{array}$ & $\begin{array}{l}-3.829 * * \\
(0.019)\end{array}$ & $\begin{array}{l}-2.377 * * \\
(0.026)\end{array}$ & $\begin{array}{l}-5.052 * * * \\
(0.004)\end{array}$ & $\begin{array}{l}-5.048^{* * *} \\
(0.004)\end{array}$ & $\begin{array}{l}-4.519 * * \\
(0.013)\end{array}$ & $\begin{array}{l}-2.174 * * \\
(0.040)\end{array}$ & $\begin{array}{l}-4.259^{* *} \\
(0.014)\end{array}$ & $\begin{array}{l}-4.298^{* *} \\
(0.014)\end{array}$ & $\begin{array}{l}-3.912^{* *} \\
(0.030)\end{array}$ \\
\hline Ln POP_j & $\begin{array}{l}0.325 * * * \\
(0.000)\end{array}$ & $\begin{array}{l}0.788^{* * *} \\
(0.000)\end{array}$ & $\begin{array}{l}0.787 * * * \\
(0.000)\end{array}$ & $\begin{array}{l}0.809^{* * *} \\
(0.000)\end{array}$ & $\begin{array}{l}0.898^{* *} \\
(0.034)\end{array}$ & $\begin{array}{l}5.758^{* * *} \\
(0.004)\end{array}$ & $\begin{array}{l}5.762 * * * \\
(0.004)\end{array}$ & $\begin{array}{l}5.280^{* *} \\
(0.014)\end{array}$ & $\begin{array}{l}0.289^{* * *} \\
(0.000)\end{array}$ & $\begin{array}{l}0.784^{* * *} \\
(0.000)\end{array}$ & $\begin{array}{l}0.785^{* * *} \\
(0.000)\end{array}$ & $\begin{array}{l}0.806^{* * *} \\
(0.000)\end{array}$ \\
\hline RISK_i & & $\begin{array}{l}0.035 \\
(0.148)\end{array}$ & $\begin{array}{l}0.037 \\
(0.129)\end{array}$ & $\begin{array}{l}0.035 \\
(0.175)\end{array}$ & & $\begin{array}{l}0.032 \\
(0.193)\end{array}$ & $\begin{array}{l}0.032 \\
(0.195)\end{array}$ & $\begin{array}{l}0.035 \\
(0.184)\end{array}$ & & $\begin{array}{l}0.029 \\
(0.221)\end{array}$ & $\begin{array}{l}0.029 \\
(0.234)\end{array}$ & $\begin{array}{l}0.032 \\
(0.197)\end{array}$ \\
\hline RISK_j & & $\begin{array}{l}0.021 \\
(0.154)\end{array}$ & $\begin{array}{l}0.022 \\
(0.146)\end{array}$ & $\begin{array}{l}0.017 \\
(0.280)\end{array}$ & & $\begin{array}{l}-0.026 \\
(0.691)\end{array}$ & $\begin{array}{l}-0.026 \\
(0.692)\end{array}$ & $\begin{array}{l}-0.033 \\
(0.637)\end{array}$ & & $\begin{array}{l}-0.004 \\
(0.907)\end{array}$ & $\begin{array}{l}-0.004 \\
(0.900)\end{array}$ & $\begin{array}{l}-0.006 \\
(0.858)\end{array}$ \\
\hline MARK_i & & $\begin{array}{l}-0.002 * \\
(0.092)\end{array}$ & $\begin{array}{l}-0.002 * \\
(0.081)\end{array}$ & $\begin{array}{l}-0.002 * \\
(0.098)\end{array}$ & & $\begin{array}{l}-0.002^{*} \\
(0.068)\end{array}$ & $\begin{array}{l}-0.002 * \\
(0.068)\end{array}$ & $\begin{array}{l}-0.002 * \\
(0.094)\end{array}$ & & $\begin{array}{l}-0.002 \\
(0.105)\end{array}$ & $\begin{array}{l}-0.002 \\
(0.113)\end{array}$ & $\begin{array}{l}-0.002 \\
(0.138)\end{array}$ \\
\hline MARK_j & & $\begin{array}{l}0.001 * * \\
(0.036)\end{array}$ & $\begin{array}{l}0.001 * * \\
(0.029)\end{array}$ & $\begin{array}{l}0.001^{* *} \\
(0.019)\end{array}$ & & $\begin{array}{l}-0.001 \\
(0.656)\end{array}$ & $\begin{array}{l}-0.001 \\
(0.661)\end{array}$ & $\begin{array}{l}-0.001 \\
(0.946)\end{array}$ & & $\begin{array}{l}-0.001 \\
(0.813)\end{array}$ & $\begin{array}{l}-0.001 \\
(0.789)\end{array}$ & $\begin{array}{l}0.001 \\
(0.886)\end{array}$ \\
\hline TRADE_ij & & & $\begin{array}{l}-1.768 \\
(0.245)\end{array}$ & & & & $\begin{array}{l}-0.139 \\
(0.976)\end{array}$ & & & & $\begin{array}{l}0.982 \\
(0.763)\end{array}$ & \\
\hline $\begin{array}{l}\text { TRADE_ij } \\
\text { (lagged) }\end{array}$ & & & & $\begin{array}{l}-0.562 \\
(0.600)\end{array}$ & & & & $\begin{array}{l}1.290 \\
(0.872)\end{array}$ & & & & $\begin{array}{l}0.178 \\
(0.923)\end{array}$ \\
\hline Ln DIST & $\begin{array}{l}-0.466^{* * *} \\
(0.000)\end{array}$ & $\begin{array}{l}-0.571^{* * *} \\
(0.000)\end{array}$ & $\begin{array}{l}-0.604 * * * \\
(0.000)\end{array}$ & $\begin{array}{l}-0.557^{* * *} \\
(0.000)\end{array}$ & & & & & $\begin{array}{l}-0.416^{* * *} \\
(0.000)\end{array}$ & $\begin{array}{l}-0.589^{* *} \\
(0.041)\end{array}$ & $\begin{array}{l}-0.569^{*} \\
(0.050)\end{array}$ & $\begin{array}{l}-0.584 * \\
(0.067)\end{array}$ \\
\hline CONTIG & $\begin{array}{l}-0.205 \\
(0.247)\end{array}$ & $\begin{array}{l}0.799 * * \\
(0.034)\end{array}$ & $\begin{array}{l}1.063 * * \\
(0.029)\end{array}$ & $\begin{array}{l}0.747^{*} \\
(0.056)\end{array}$ & & & & & $\begin{array}{l}0.348 \\
(0.375)\end{array}$ & $\begin{array}{l}0.681 \\
(0.441)\end{array}$ & $\begin{array}{l}0.578 \\
(0.548)\end{array}$ & $\begin{array}{l}0.630 \\
(0.486)\end{array}$ \\
\hline COMLANG & $\begin{array}{l}0.131 \\
(0.178)\end{array}$ & $\begin{array}{l}0.580^{* * * *} \\
(0.004)\end{array}$ & $\begin{array}{l}0.583^{* * *} \\
(0.003)\end{array}$ & $\begin{array}{l}0.656^{* * * *} \\
(0.004)\end{array}$ & & & & & $\begin{array}{l}0.195 \\
(0.363)\end{array}$ & $\begin{array}{l}0.576 \\
(0.205)\end{array}$ & $\begin{array}{l}0.571 \\
(0.209)\end{array}$ & $\begin{array}{l}0.548 \\
(0.275)\end{array}$ \\
\hline CONSTANT & $\begin{array}{l}28.372 * \\
(0.051)\end{array}$ & $\begin{array}{l}30.129 \\
(0.335)\end{array}$ & $\begin{array}{l}28.867 \\
(0.345)\end{array}$ & $\begin{array}{l}50.455 \\
(0.191)\end{array}$ & $\begin{array}{l}-14.390 \\
(0.442)\end{array}$ & $\begin{array}{l}-18.625 \\
(0.698)\end{array}$ & $\begin{array}{l}-18.876 \\
(0.703)\end{array}$ & $\begin{array}{l}-23.310 \\
(0.654)\end{array}$ & $\begin{array}{l}28.357 \\
(0.146)\end{array}$ & $\begin{array}{l}58.074 * \\
(0.093)\end{array}$ & $\begin{array}{l}58.863^{*} \\
(0.090)\end{array}$ & $\begin{array}{l}51.875 \\
(0.150)\end{array}$ \\
\hline $\begin{array}{l}\text { Source country } \\
\text { year effects }\end{array}$ & Yes & Yes & Yes & Yes & Yes & Yes & Yes & Yes & Yes & Yes & Yes & Yes \\
\hline $\begin{array}{l}\text { Number of } \\
\text { Observations }\end{array}$ & 3530 & 838 & 838 & 794 & 3530 & 838 & 838 & 794 & 3530 & 838 & 838 & 794 \\
\hline $\begin{array}{l}\text { Number of } \\
\text { Country }\end{array}$ & 375 & 115 & 115 & 109 & 375 & 115 & 115 & 109 & 375 & 115 & 115 & 109 \\
\hline $\mathrm{R}^{2}$ (within) & 0.6286 & 0.7736 & 0.7740 & 0.7682 & 0.3432 & 0.4293 & 0.4293 & 0.4253 & 0.3416 & 0.4095 & 0.4097 & 0.4087 \\
\hline $\mathrm{R}^{2}$ (between) & & & & & 0.1060 & 0.1624 & 0.1624 & 0.1543 & 0.7162 & 0.8115 & 0.8114 & 0.8046 \\
\hline $\mathrm{R}^{2}$ (overall) & & & & & 0.1333 & 0.1689 & 0.1690 & 0.1552 & 0.6260 & 0.7639 & 0.7633 & 0.7589 \\
\hline
\end{tabular}

Note. subscript "i”" stands for source economy and " $\mathrm{j}$ ” for destination economy.

p-value in parentheses, *significant at $10 \%$, **significant at 5\%, ***significant at $1 \%$. Note: Ln (natural logarithm form).

In relation to the control variables, it was observed that the risk premium on lending turned to a positive coefficient and significance for source economies for OLS (models 2, 3 and 4), FE (models 7 and 8), and RE (models 1011 , and 12), consistent with Hattari and Rajan (2011). The $\mathrm{R}^{2}$ indicated that the models explained in OLS estimations 59 per cent of the variation in portfolio investment in model 1 and 75 per cent of the variation in portfolio investment in models 2, 3, and 4.

\subsection{Determinants of Cross-Border Portfolio Investment using Source Country Year Effects}

It was also interesting to assess that these estimations differ from the previous regressions by involving the year-specific effects and source country-specific effects; Table 5 reported the results for equation (3). Turning to the major issue of the effect of GDP per capita in source countries and destination country on assets, GDP per capita in the source country, unexpectedly, insignificantly affects assets as evidenced in all three methods. On the other hand, the coefficients of GDP per capita in the destination country are positive and statistically highly 
significant in OLS (models 1 to 4), and RE (models 9 to 12) as expected. The estimation of the cross-section models of per capita GDP levels is performed for all the factors. Comparing OLS and RE, there is a negative (unexpected) coefficient for models in both cases as it is not statistically significant.

\section{Discussion}

The results have shown positive and significant impact on GDP per capita in the destination country for OLS (models 1 to 4), FE (only model 5), and RE (models 9 to 12). These findings suggested that richer economies are major recipients of investment holding. These findings broadly provide support for a number of studies (Porter \& Rey, 2005; Lee et al., 2012). The larger economies are major sources and recipients of investment holdings, consistent with the empirical evidence (Papaioannou, 2009; Peter, 2012). Moreover, the distance in the determinants portfolio investment become insignificant when trade was added as an independent variable, which revealed that distance may not directly influence financial flows (Shin \& Yang, 2006). Similarly, Hutchinson (2002) utilized a gravity model between the United States and other 33 countries to determine the impact of the degree of language commonality on bilateral trade. The results clearly depicted that GDP per capita and distance was negative and significant. These results were consistent with resultes deduced in the present study.

The results of this study suggest that geographical proximity exerts a significant positive influence on the assets that investors may diversify their portfolios. Furthermore, the study conducted by Jagannathan, Jiao, and Karolyi (2017) showed that managers hired by international equity mutual funds from a country is linked to the fund's geographic mandate, which shows a limitation for the investment in stocks of that country. This finding may be related to the source country and ought to lead to greater outflows due to the issue of an uncertainty aversion and sunk costs. This finding has been supported and suggested that the relative risk of incurring sunk costs overseas was lower (Hattari \& Rajan, 2011). The estimates also showed that contiguous aspects had a positive and significant affects only in OLS (models 2, 3 and 4). These results were in line with Hahm and Shin (2009). These results confirmed that information asymmetry was an important determinant of cross-border asset holdings, which are concerned with the power of the gravity model. This could be attributed to the possibility that investors in developing countries may tend to invest more with countries that share a border and have the same language.

Moreover, the study conducted by Camanho, Hau, and Rey (2018) showed that the institutional investors after earning an excess amount of their portfolio share their capital. The study showed that there are many reasons behind the rebalancing and also suggested some recommendations to solve the issue of rebalancing. Bertay et al. (2016) conducted a study to evaluate cross-border banking from the financial safety net. The study was conducted on the bank-level data of 84 countries and the results of the study showed that a high level of bank internationalization is related with higher interest expenses. Moreover, the study suggested that if the bank is headquartered in a country with weak public finances or if the bank is underperforming then its interest expenses rises at a great rate. Similar to the findings of present study, Ramaswamy et al. (2016) analysed the performance of free trade agreements by evaluating the constraints of trade flows of Asian economies from 2007-2014, using gravity model. The results depicted a negative effect of distance between the countries involved in the trade, suggesting that GDP and population explains the total trade flow.

\section{Conclusion}

The study has found that GDP per capita is positively affected for source and destination economies; whereas, population has a negative effect for source economies. Risk premium in source economies had a positive effect; whilst market capitalization of listed companies in source economies had a negative effect. The results showed no statistically significant effect of bilateral trade in lagged form on asset holdings. The findings had two main policy implications. Firstly, Lucas paradox supported the evidence that developing investors preferred to invest outside the region instead of in developing markets. Secondly, they preferred to buy equities in distant countries for optimal diversification. For future research, it would be interesting to combine three main results, checking which effect is predominant in the decision to invest.

\section{Acknowledgements}

The author is very thankful to all the associated personnel in any reference that contributed in/for the purpose of this research.

\section{References}

Abid, F., \& Bahloul, S. (2011). Selected MENA countries' attractiveness to G7 investors. Economic Modelling, 28(5), 2197-2207. https://doi.org/10.1016/j.econmod.2011.06.013

Abidin, I. S. Z., \& Sahlan, R. (2013). The determinants of exports between Malaysia and the OIC member 
countries: A gravity model approach. Procedia Economics and Finance, 5, 12-19. https://doi.org/10.1016/S2212-5671(13)00004-X

Aggarwal, R., Kearney, C., \& Lucey, B. (2012). Gravity and culture in foreign portfolio investment. Journal of Banking \& Finance, 36(2), 525-538. https://doi.org/10.1016/j.jbankfin.2011.08.007

Alfaro, L., \& Chauvin, J. (2016). Foreign Direct Investment, Finance, and Economic Development.

Ashraf, A., Herzer, D., \& Nunnenkamp, P. (2016). The effects of Greenfield FDI and cross - border M\&As on total factor productivity. The World Economy, 39(11), 1728-1755. https://doi.org/10.1111/twec.12321

Aviat, A., \& Coeurdacier, N. (2007). The geography of trade in goods and asset holdings. Journal of International Economics, 71(1), 22-51. https://doi.org/10.1016/j.jinteco.2006.02.002

Balli, F., Louis, R. J., \& Osman, M. (2011). The patterns of cross-border portfolio investments in the GCC region: do institutional quality and the number of expatriates play a role?. Journal of Economics and Finance, 35(4), 434-455. https://doi.org/10.1007/s12197-009-9111-5

Bank for International Settlements. (2010). Long-Term Issues in International Banking.

Bertay, A. C., Demirgüç-Kunt, A., \& Huizinga, H. (2016). Should cross-border banking benefit from the financial safety net?. Journal of Financial Intermediation, 27, 51-67. https://doi.org/10.1016/j.jfi.2016.05.005

Birula, J.B., Modelling International Trade in Art - Modified Gravity Approach. Procedia Economics and Finance, 30, 91-99. 2015. https://doi.org/10.1016/S2212-5671(15)01258-7

Bruno, V., \& Shin, H. S. (2014). Cross-border banking and global liquidity. The Review of Economic Studies, 82(2), 535-564. https://doi.org/10.1093/restud/rdu042

Choi, C., Rhee, D. E., \& Oh, Y. (2014). Information and capital flows revisited: The Internet as a determinant of transactions in financial assets. Economic Modelling, 40, 191-198. https://doi.org/10.1016/j.econmod.2014.03.027

Coeurdacier, N., \& Martin, P. (2009). The geography of asset trade and the euro: Insiders and outsiders. Journal of the Japanese and International Economies, 23(2), 90-113. https://doi.org/10.1016/j.jjie.2008.11.001

Coeurdacier, N., \& Rey, H. (2013). Home bias in open economy financial macroeconomics. Journal of Economic Literature, 51(1), 63-115. https://doi.org/10.1257/jel.51.1.63

Daly, K., \& Vo, X.V. (2013). The determinants of home bias puzzle in equity portfolio investment in Australia. International Review of Financial Analysis, 27, 34-42. https://doi.org/10.1016/j.irfa.2012.05.005

Elshehawy, M. A., Shen, H., \& Ahmed, R. A. (2014). The factors affecting Egypt's exports: Evidence from the gravity model analysis. Open Journal of Social Sciences, 2(11), 138. https://doi.org/10.4236/jss.2014.211020

García-Herrero, A., Wooldridge, P., \& Yang, D. Y. (2009). Why don't Asians invest in Asia? The determinants of cross-border portfolio holdings. Asian Economic Papers, 8(3), 228-246. https://doi.org/10.1162/asep.2009.8.3.228

Hahm, J. H., \& Shin, K. (2009). Complementarity among international asset holdings. Journal of the Japanese and International Economies, 23(1), 37-55. https://doi.org/10.1016/j.jjie.2009.01.001

Hattari, R., \& Rajan, R. S. (2011). How different are FDI and FPI flows?: Distance and capital market integration. Journal of Economic Integration, 499-525. https://doi.org/10.11130/jei.2011.26.3.499

Hutchinson, W.K., Does Ease of Communication Increase Trade? Commonality of Language and Bilateral Trade. Scottish Journal of Political Economy, 49. 2002. https://doi.org/10.1111/1467-9485.00247

Jagannathan, M., Jiao, W., \& Karolyi, G. (2017). Is There a Home Field Advantage in Global Markets?.

Julio, B., \& Yook, Y. (2016). Policy uncertainty, irreversibility, and cross-border flows of capital. Journal of International Economics, 103, 13-26. https://doi.org/10.1016/j.jinteco.2016.08.004

Lee, H. H., Huh, H. S., \& Kim, W. J. (2012). Cross-border portfolio investment in the APEC region. Japan and the World Economy, 24(1), 44-56. https://doi.org/10.1016/j.japwor.2011.11.002

Lucas, R. E. (1990). Why doesn't capital flow from rich to poor countries?. The American Economic Review, 80(2), 92-96.

Martin, P., \& Rey, H. (2004). Financial super-markets: size matters for asset trade. Journal of International 
Economics, 64(2), 335-361. https://doi.org/10.1016/j.jinteco.2003.12.001

Moshirian, F., Pham, P., Tian, S., \& Wu, E. (2017). Economic Integration Breeds Foreign Investment: Evidence from the Portfolio Allocation of Investment Funds around the World.

Narula, R., \& Pineli, A. (2017). Multinational enterprises and economic development in host countries: What we know and what we don't know. In Development Finance (pp. 147-188). Palgrave Macmillan, London. https://doi.org/10.1057/978-1-137-58032-0_6

Papaioannou, E. (2009). What drives international financial flows? Politics, institutions and other determinants. Journal of Development economics, 88(2), 269-281. https://doi.org/10.1016/j.jdeveco.2008.04.001

Peter, A. (2012). Bilateral trade, openness, and asset holdings. Open Economies Review, 23(4), 713-740. https://doi.org/10.1007/s11079-011-9211-7

Portes, R., \& Rey, H. (2005). The determinants of cross-border equity flows. Journal of international Economics, 65(2), 269-296. https://doi.org/10.1016/j.jinteco.2004.05.002

Portes, R., Rey, H., \& Oh, Y. (2001). Information and capital flows: The determinants of transactions in financial assets. European Economic Review, 45(4-6), 783-796. https://doi.org/10.1016/S0014-2921(01)00138-6

Ramaswamy, S., Choutagunta, A., \& Sahu, S. K. (2016). Evaluating Asian FTAs: What do Gravity Equation Models Tell Us? (No. 2016-152).

Shin, K., \& Yang, D. Y. (2012). Complementarities between bilateral trade and financial integration. Korea and the World Economy, 13(1), 39-68.

\section{Appendix}

\section{Appendix A}

Table A1. List of source countries (37 developing countries)

\begin{tabular}{cccc}
\hline Argentina & Colombia & Lebanon & Romania \\
Aruba & Costa Rica & Macau, China & Russia \\
Bahrain & Egypt & Malaysia & Singapore \\
Barbados & Hong Kong, China & Mauritius & South Africa \\
Bermuda & India & Mexico & Thailand \\
Bolivia & Indonesia & Mongolia & Turkey \\
Brazil & Kazakhstan & Netherlands Antilles & Ukraine \\
Bulgaria & Korea & Pakistan & Uruguay \\
Chile & Kuwait & Philippines & Vanuatu \\
& & & Venezuela \\
\hline
\end{tabular}

Table A2. List of 79 host countries

\begin{tabular}{|c|c|c|c|c|}
\hline Spain & Moldova & Hong Kong & Chile & Australia \\
\hline Qatar & Netherlands Antilles & Hungary & Colombia & Austria \\
\hline Taiwan & Netherlands & India & Canada & Argentina \\
\hline Thailand & New Zealand & Israel & Croatia & Brazil \\
\hline Turkey & Nicaragua & Italy & Central African & Belize \\
\hline Trinidad and Tobago & Panama & Japan & Republic & Belarus \\
\hline Ukraine & Philippines & Jamaica & Denmark & Bangladesh \\
\hline United States & Portugal & Jersey & Ireland & Belgium \\
\hline United Kingdom & Russian & Jordan & Indonesia & Bahamas \\
\hline United Arab Emirates & Saudi Arabia & Korea & El Salvador & Bahrain \\
\hline Uruguay & Sweden & Luxembourg & Egypt & Bermuda \\
\hline Virgin Islands, Britsh & Switzerland & Latvia & France & Curacao \\
\hline \multirow[t]{5}{*}{ Virgin Islands,US } & Singapore & Mexico & Finland & Cyprus \\
\hline & Sri Lanka & Mauritius & Germany & Cuba \\
\hline & Swaziland & Macedonia & Greenland & Cayman Islands \\
\hline & South Africa & Madagascar & Guatemala & Czech Republic \\
\hline & & Malaysia & Greece & China \\
\hline
\end{tabular}


Table A3. Variables and definitions

\begin{tabular}{|c|c|}
\hline Variables & Definitions \\
\hline Dependent Variable & bilateral cross-border assets holding (equity securities and debt securities in short term and long term) \\
\hline $\mathrm{FPI}_{\mathrm{ij}}$ & between source country $i$ and host country $j$ \\
\hline Explanatory Variables & Real GDP per capita (constant 2005US) in sources country i \\
\hline \multicolumn{2}{|l|}{ GDP pc_i } \\
\hline Ln GDP pc_i & Real GDP per capita (constant 2005US) in sources country i, in natural logarithm form \\
\hline GDP pc $\_j$ & Real GDP per capita (constant 2005US) in host country j \\
\hline LnGDP pc $\_j$ & Real GDP per capita (constant 2005US) in host country $\mathrm{j}$, in natural logarithm form \\
\hline POP_i & Population in sources country $i$ \\
\hline Ln POP_i & Population in sources country $i$, in natural logarithm form \\
\hline $\mathrm{POP}_{-} \mathrm{j}$ & Population in host country $\mathrm{j}$ \\
\hline Ln POP_j & Population in host country $\mathrm{j}$, in natural logarithm form \\
\hline RISK_i & Risk premium on lending in source country $\mathrm{i}$ (prime rate minus treasury bill rate, $\%$ ) \\
\hline RISK_j & Risk premium on lending in host country $\mathrm{j}$ (prime rate minus treasury bill rate, $\%$ ) \\
\hline MARK_i & Market capitalization of listed companies in source country i (\% of GDP) \\
\hline MARK $\mathrm{j}$ & Market capitalization of listed companies in host country $\mathrm{j}$ (\% of GDP) \\
\hline TRADE_ij & $\begin{array}{l}\text { Total ratio of bilateral trade (exports + imports) between source and destination countries relative to the } \\
\text { destination country's GDP }\end{array}$ \\
\hline TRADE_ij (lagged) & $\begin{array}{l}\text { Total ratio of bilateral trade (exports }+ \text { imports) between source and destination countries relative to the } \\
\text { destination country's GDP (one-year lagged) }\end{array}$ \\
\hline Explanatory Variables & The geographical distances (miles) between source and host countries. \\
\hline \multicolumn{2}{|l|}{ DIST $_{\mathrm{ij}}$} \\
\hline $\operatorname{Ln~DIST}_{\mathrm{ij}}$ & The geographical distances (miles) between source and host countries in natural logarithm form. \\
\hline CONTIG & $\begin{array}{l}\text { Dummy variable, which equals one if an origin/ destination country pair shares an official language, and } \\
\text { zero otherwise. }\end{array}$ \\
\hline COMLANG & Dummy variable, which equals one if an origin, destination country pair shares a border, and zero otherwise. \\
\hline
\end{tabular}

Sources: Data for international portfolio investment (2001-2012) are obtained from the International Monetary Fund's (IMF) coordinated Portfolio Investment Survey (CIPS).

Data for GDP pc, Population, Risk, and Market capitalization (2001-2012) from World Development Indicator (December 2014).

Bilateral trade (2001-2012), Bilateral exports and imports from International Monetary Fund, Direction of Trade Statistics, and GDP destination country's data is taken from the World Development Indicators Database (World Bank, 2014).

Distance, Contiguous, and common an official language (2001-2012) from Centre d' Etudes Prospective et d' Information's Internationals (CEPII)'s.

\section{Copyrights}

Copyright for this article is retained by the author(s), with first publication rights granted to the journal.

This is an open-access article distributed under the terms and conditions of the Creative Commons Attribution license (http://creativecommons.org/licenses/by/4.0/). 